\title{
Detection of influenza B lineages from 2001 to 2013 in a tertiary hospital in the city of São Paulo, Brazil
}

\author{
Ana Helena Perosa $/{ }^{+}$, Celso Granato, Nancy Bellei \\ Universidade Federal de São Paulo, Departamento de Medicina, Laboratório de Virologia Clínica, Disciplina de Infectologia, São Paulo, SP, Brasil
}

Two antigenically distinct lineages of influenza B viruses, the Victoria-like and Yamagata-like strains, currently circulate among humans. Surveillance from United States of America and Europe over the last 10 years showed that the chance of a correct matching between vaccine and the circulating lineage had been $50 \%$. We investigated influenza B infection in different patient groups (asymptomatic, general community, with comorbidities and hospitalised) attended at a tertiary hospital in the city of São Paulo, Brazil between 2001-2013. All samples were screened for influenza B virus by one-step real-time reverse transcription-polymerase chain reaction. From 2,992 respiratory samples collected, 114 (3.8\%) tested positive for influenza B. Teenagers (13-18 years) presented the highest rate of $18.5 \%$ (odds ratio $22.87,95 \%$ confidence interval 2.90-180.66, $p<0.001$ ). One hundred nine samples could be characterised: 50 were Yamagata-like and 59 were Victoria-like strains. Mismatching between the vaccine and predominant circulating strain was observed in 2002 and 2013 seasons. Based on data collected during a period of 12 years, we found that influenza $B$ was more frequent in teenagers. Co-circulation of both lineages and mismatch with the vaccine strain can occur. Our data highlighted the importance of quadrivalent vaccines and future analysis of the age groups included in vaccination programs.

Key words: influenza B lineages - influenza vaccine - epidemiology - respiratory infection

Influenza infections in humans can be caused by type $\mathrm{A}$ and type $\mathrm{B}$ viruses. Influenza $\mathrm{A}$ is responsible for the pandemics and the majority of seasonal infections and influenza B incidence can vary between seasons. Influenza $\mathrm{B}$ infections are common in children and young adults and the rates of hospitalisations, pneumonia and deaths among children infected with influenza B are similar to those for influenza A (Gutiérrez-Pizarraya et al. 2012).

Influenza $\mathrm{B}$ can be classified into two antigenically distinct lineages, B/Victoria/02/87-like and B/ Yamagata/16/88-like, based on divergence in the HA1 domain of the viral haemagglutinin gene, and this difference is responsible for the lack of antigenic cross reactivity between lineages. Reference laboratories have differentiated the lineages by haemagglutination-inhibition testing, which is a time-consuming technique that requires virus isolation. These two lineages currently circulate among humans since 1983 (Rota et al. 1990). Influenza $\mathrm{B}$ evolution is characterised by a continuous turnover of lineages and the match between circulating and vaccine strains vary annually (Chen \& Holmes 2008). In Brazil, the current influenza vaccine distributed by the National Immunisation Program to high-risk groups for influenza complications contains only one influenza B lineage.

doi: 10.1590/0074-02760150044

Financial support: FAPESP (2013/00715-0), CNPq

+ Corresponding author: anaperosa@gmail.com

Received 30 January 2015

Accepted 3 June 2015
Surveillance from 2001-2011 in the United States of America and Europe showed that the chance of a correct matching between vaccine and circulating influenza B lineages was 50\% (Ambrose \& Levin 2012). Consequently, the influenza vaccination was less effective during mismatched seasons. Information about the circulation of these lineages is important to assess the need for a quadrivalent influenza vaccine. The objective of this study was to describe the influenza B circulation in different patient groups from a tertiary hospital of the largest city in the Southern Hemisphere from 2001-2013 and the lineage differentiation of this virus using a sensitive and lineage specific real-time reverse transcriptionpolymerase chain reaction (RT-PCR) method.

\section{SUBJECTS, MATERIALS AND METHODS}

Study design and sample collection - During the 12-year study, respiratory samples were collected from subjects with different risk for influenza acquisition. We evaluated 2,992 samples (1,926 nasopharyngeal swabs, 871 nasal washes and 195 nasopharyngeal aspirates) collected between June 2001-October 2013 (except 2006) from patients attended at primary care health service in ambulatory or specific units of a tertiary hospital (a referral hospital with basic services and specific care) from the city of São Paulo, Brazil. Each of these health services had a variable flow of sampling, which resulted in collections of different patient groups, unevenly distributed throughout the study period. Samples were classified into six patients groups: (i) emergency care: outpatients from the general community (adults, children and healthcare workers) with acute respiratory infection (ARI), collected from 2001-2005 and 2008-2013; (ii) kidney transplant (KT) recipients: adults outpatients with ARI recruited during routine visits, collected from 
2002-2004; (iii) high-risk children: children with heart and/or lung diseases and/or premature birth with ARI recruited during routine visits, collected from 2005-2008; (iv) human immunodeficiency virus (HIV)-positive patients: adults outpatients with ARI or asymptomatic recruited once a week during scheduled routine visits during influenza epidemic period, collected from 2010-2013; (v) patients in a haematopoietic stem cell transplant (HSCT) program: hospitalised adults with ARI or contact with a symptomatic patient, collected from 2004-2013; (vi) hospitalised patients: inpatients (adults and children) with severe acute respiratory infection (SARI), suspected of 2009 H1N1 influenza A infection, collected from 2009 to 2013.

Laboratory methods - Nucleic acid was extracted from $140 \mu \mathrm{L}$ aliquots that had been stored at $-80^{\circ} \mathrm{C}$, using the QIAmp Viral RNA Extraction Kit (Qiagen, Germany), according to the manufacturer's recommendations. The influenza B one-step real-time RT-PCR was performed using primers for the nonstructural gene (forward: 5'-TCCTCAACTCACTCTTCGAGCG-3'; reverse: 5'-CGGTGCTCTTGACCAAATTGG-3'; probe: 5'-VICCCAATTCGAGCAGCTGAAACTGCGGTG-MGB-3') previously described by Selvaraju and Selvarangan (2010). Reaction mixtures $(25 \mu \mathrm{L})$ were prepared using the AgPath-ID ${ }^{\mathrm{TM}}$ One-Step RT-PCR Kit (Ambion, USA) and contained final concentrations: 1x RT- PCR buffer, 1x RTPCR enzyme mix, $0.8 \mu \mathrm{M}$ of each primer, $0.2 \mu \mathrm{M}$ probe, nuclease-free water and $5 \mu \mathrm{L}$ of nucleic acid. Amplification was performed on ABI 7500 Real-time PCR System (Applied Biosystems, USA) with the following cycling conditions: $50^{\circ} \mathrm{C}$ for $10 \mathrm{~min}, 95^{\circ} \mathrm{C}$ for $10 \mathrm{~min}$ and $95^{\circ} \mathrm{C}$ for $15 \mathrm{~s}$ followed by $55^{\circ} \mathrm{C}$ for $30 \mathrm{~s}$ ( $45 \mathrm{cycles}$ ). Each specimen was also tested for the human ribonuclease $\mathrm{P}$ gene (constitutive gene) as an internal control (CDC 2009).

Influenza $\mathrm{B}$ positive samples were tested to identify the lineages using a single one step real-time RT-PCR with one pair of primers (forward: 5'- ACCCTACAR-
AMTTGGAACYTCAGG-3'; reverse: 5'-ACAGCCCAAGCCATTGTTG-3') and two probes with different colour labels: 5'-FAM-AATCCGMTYTTACTGGTAGMGB-3' (for identification of Yamagata lineage) and 5'VIC-ATCCGTTTCCATTGGTAA-MGB-3' (for identification of Victoria lineage) previously described by Biere et al. (2010). Reaction mixtures $(25 \mu \mathrm{L})$ were prepared using the AgPath-ID ${ }^{\mathrm{TM}}$ One-Step RT-PCR Kit (Ambion) and contained final concentrations: 1x RT-PCR buffer, 1x RT-PCR enzyme mix, $0.9 \mu \mathrm{M}$ of forward primer, 0.6 $\mu \mathrm{M}$ of reverse primer, $0.15 \mu \mathrm{M}$ of each probe, nucleasefree water and $5 \mu \mathrm{L}$ of nucleic acid. Amplification was performed on ABI 7500 Real-time PCR System (Applied Biosystems) with the following cycling conditions: $50^{\circ} \mathrm{C}$ for $10 \mathrm{~min}, 95^{\circ} \mathrm{C}$ for $10 \mathrm{~min}$ and $95^{\circ} \mathrm{C}$ for $15 \mathrm{~s}$ followed by $60^{\circ} \mathrm{C}$ for $30 \mathrm{~s}$ (50 cycles).

Data analysis - We considered as "predominated year" when Victoria or Yamagata lineages circulated in over $80 \%$ of the specimens. Additionally, each influenza season was categorised as matched or mismatched after comparing the most commonly circulating influenza B lineage and the vaccine component distributed by the Brazilian Health Office.

Statistical analysis consisted of chi-squared test for the comparison of categorical values, with a significance level of $p<0.05$. Results are presented as odds ratios and the respective $95 \%$ confidence intervals (CI). All data were analysed using the Statistical Package for Social Sciences v.18.0.0 (SPSS IBM).

\section{RESULTS}

We analysed 2,992 respiratory samples and the median age of all studied patients was $28 \pm 22$ years (range: 14 days-91 years); 284 (9.5\%) patients had been vaccinated against influenza in the same year of sample collection. The median age and standard deviation of each patient group was: 24 years $( \pm 18)$ for emergency care,

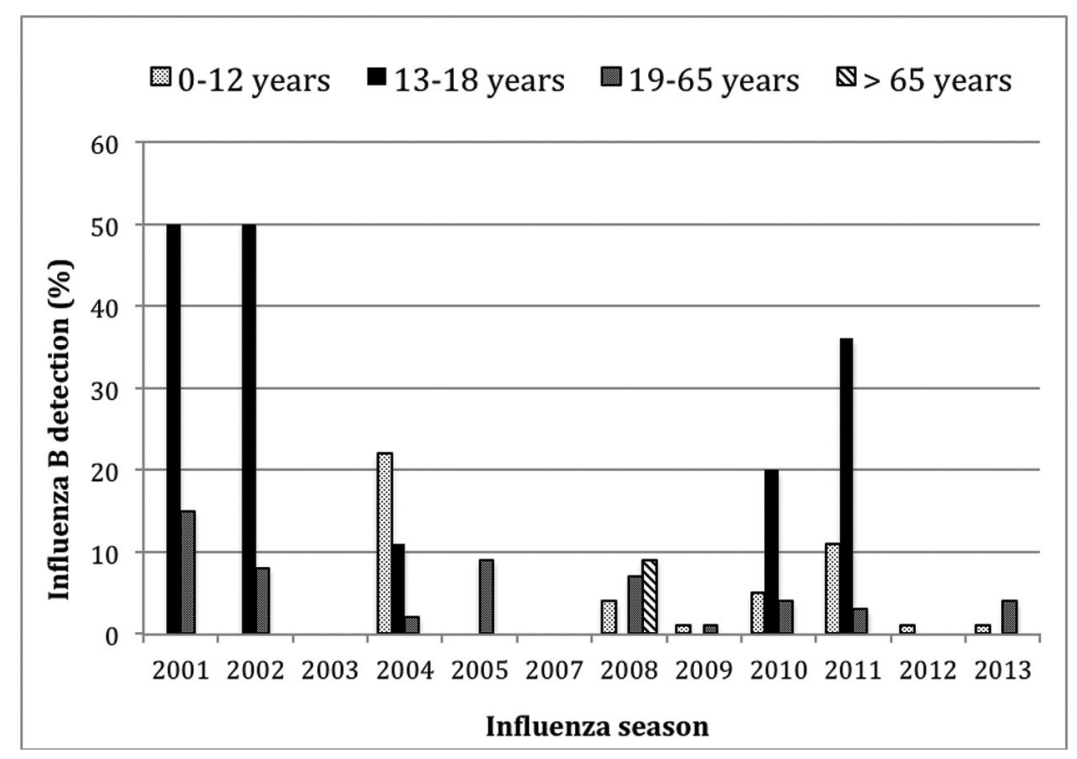

Influenza B detection in different age groups by influenza season (there was no sampling during 2006). 
TABLE I

Demographic and epidemiological characteristics of subjects

\begin{tabular}{|c|c|c|c|c|}
\hline & $\begin{array}{c}\text { Samples tested } \\
\mathrm{n}\end{array}$ & $\begin{array}{c}\text { Influenza B } \\
\mathrm{n}(\%)\end{array}$ & $\mathrm{p}$ & $\begin{array}{l}\text { Odds ratio } \\
(95 \% \mathrm{CI})\end{array}$ \\
\hline \multicolumn{5}{|l|}{ Patient groups } \\
\hline Emergency care & 1,468 & $76(5.2)$ & $0.002^{a}$ & $10.59(1.47-76.60)^{a}$ \\
\hline $\mathrm{KT}$ & 251 & $10(4)$ & & $8.0(1.02-63.17)^{a}$ \\
\hline HSCT & 255 & $7(2.7)$ & & $5.48(0.67-44.88)$ \\
\hline HIV & 139 & $2(1.4)$ & & $2.83(0.25-31.55)$ \\
\hline Hospitalised & 684 & $18(2.6)$ & & $5.24(0.70-39.53)$ \\
\hline High-risk children & 195 & $1(0.5)$ & & 1 (Ref) \\
\hline \multicolumn{5}{|l|}{ Vaccine } \\
\hline Yes & 284 & $8(2.8)$ & 0.359 & - \\
\hline No or not informed & 2,708 & $106(3.9)$ & & \\
\hline \multicolumn{5}{|l|}{ Symptoms } \\
\hline ARI & 1,908 & $93(4.9)$ & $<0.001^{a}$ & $6.78(2.14-21.52)^{a}$ \\
\hline SARI & 684 & $18(2.6)$ & & $3.58(1.05-12.22)^{a}$ \\
\hline Asymptomatic & 400 & $3(0.75)$ & & 1 (Ref) \\
\hline
\end{tabular}

$a$ : significant at $\mathrm{p}<0.05$; ARI: acute respiratory infection; CI: confidence interval; HIV: human immunodeficiency virus (positive patients); HSCT: haematopoietic stem cell transplant patients; KT: kidney transplant; SARI: severe ARI.

TABLE II

Influenza B detection by age groups

\begin{tabular}{lccc}
\hline $\begin{array}{l}\text { Age groups } \\
\text { (years) }\end{array}$ & $\begin{array}{c}\text { Samples tested } \\
\mathrm{n}\end{array}$ & $\begin{array}{c}\text { Influenza B } \\
\mathrm{n}(\%)\end{array}$ & $\begin{array}{c}\text { Odds ratio } \\
(95 \% \mathrm{CI})\end{array}$ \\
\hline $0-12$ & 1,075 & $37(3.4)$ & $3.60(0.49-26.52)$ \\
$13-18$ & 65 & $12(18.5)$ & $22.87(2.90-180.66)^{a}$ \\
$19-65$ & 1,750 & $64(3.6)$ & $3.8(0.53-27.92)$ \\
$>65$ & 102 & $1(1)$ & $1($ Ref $)$
\end{tabular}

$a$ : significant at $\mathrm{p}<0.05 ; \mathrm{CI}$ : confidence interval; $\mathrm{p}:<0.001$.

TABLE III

Influenza B detection and lineage identification by year

\begin{tabular}{|c|c|c|c|c|c|}
\hline Year $^{a}$ & $\begin{array}{l}\text { Samples tested } \\
\quad(\mathrm{n}=2,992)\end{array}$ & $\begin{array}{c}\text { Influenza } \mathrm{B}^{+} \\
\mathrm{n}(\%)\end{array}$ & $\begin{array}{c}\text { Yamagata-like } \\
\text { n (\%) }\end{array}$ & $\begin{array}{c}\text { Victoria-like } \\
\text { n }(\%)\end{array}$ & Vaccine lineage $^{b}$ \\
\hline 2001 & 74 & $12(16.2)$ & $11(100)$ & - & Yamagata \\
\hline 2002 & 212 & $21(9.9)$ & $5(25)$ & $15(75)$ & Yamagata \\
\hline 2003 & 130 & 0 & - & - & Victoria \\
\hline 2004 & 190 & $7(3.7)$ & - & $6(100)$ & Victoria \\
\hline 2005 & 73 & $1(1.4)$ & $1(100)$ & - & Yamagata \\
\hline 2007 & 32 & 0 & - & - & Victoria \\
\hline 2008 & 370 & $17(4.6)$ & $9(56)$ & $7(44)$ & Yamagata \\
\hline 2009 & 586 & $5(0.8)$ & $5(100)$ & - & Yamagata \\
\hline 2010 & 438 & $20(4.7)$ & $19(95)$ & $1(5)$ & - \\
\hline 2011 & 435 & $24(5.5)$ & - & $24(100)$ & Victoria \\
\hline 2012 & 207 & $1(0.5)$ & - & $1(100)$ & Victoria \\
\hline 2013 & 245 & $6(2.4)$ & - & $5(100)$ & Yamagata \\
\hline
\end{tabular}

$a$ : there was no sampling during 2006; $b$ : vaccine recommendation for Southern Hemisphere. Vaccine distributed in Brazil during 2010 contained only the influenza A(H1N1)pdm09 strain. 
39 years $( \pm 13)$ for KT patients, 44 years $( \pm 16)$ for HSCT patients, 46 years $( \pm 10)$ for HIV-positive patients, 24 ( \pm 25 ) for hospitalised patients and one year $( \pm 3)$ for highrisk children. The demographic and epidemiological characteristics are shown in Table I.

Influenza B was detected in 114 samples (3.8\%). Influenza B detection rate was different between patients groups $(p=0.002)$ and frequency in symptomatic subjects was higher than in asymptomatic $(p<0.001)$. Vaccine was not a protective factor $(\mathrm{p}=0.359)$ (Table I).

The median age of influenza $B$ infected patients was 21 years (range, 9 months- 72 years). Only $1 \%$ of the subjects showed positivity for influenza $\mathrm{B}$ in the elderly group ( $>65$ years), whereas $18.5 \%$ of teenagers $(95 \%$ CI 2.90 $180.66)$ were positive for influenza B $(p<0.001)$. These results show a 23 -fold increase of the risk to undergo influenza B infection in teenagers (13-18 years) than in the elderly (Table II). Figure illustrates the influenza B detection rate in different age groups by influenza season.

The influenza B annual frequency ranged from $0.5 \%$ (2012) to $16.2 \%$ (2001); virus was not detected in both 2003 and 2007 (Table III). One hundred twelve influenza B samples were available for lineage differentiation and 109 samples could be characterised: 50 were Yamagata-like and 59 were Victoria-like. Eight seasons were "predominated" by one lineage: four seasons by Yamagata-like strains (2001, 2005, 2009 and 2010) and four by Victoria-like strains (2004, 2011, 2012 and 2013) and 2002 and 2008 were "mixed" seasons. Mismatching between vaccine and the most commonly detected lineage was observed in two seasons: 2002 and 2013 (Table III). Overall, 26\% (28/109) of influenza B samples were characterised as a lineage not included in the vaccine that was distributed in Brazil in the same year.

\section{DISCUSSION}

In this study, we investigated the frequency of influenza B infection in 2,992 respiratory samples collected from 2001-2013. The overall detection of influenza B was $3.8 \%$ and was higher than the $1.5 \%$ positivity rate described from 2000-2010 by the Brazilian National Surveillance (Freitas 2013); however, we used real-time PCR instead of immunofluorescence.

The majority of influenza B virus infection affected teenagers between 13-18 years, which was similar to other Brazilian study (Paiva et al. 2013). In a New York study, influenza B was more frequent in those aged five-17 years (Olson et al. 2007). During a 10-year study in Hong Kong, hospitalisation rate due to influenza B was highest among older children and young teenagers (Chan et al. 2013). Hite et al. (2007) demonstrated that children with influenza B were on average two years older when comparing to influenza A cases and the majority of children with laboratory-confirmed influenza were over two years of age. In our study, the median age of high-risk children was one year, which could explain our low influenza B detection is this group.

Influenza B was detected among all patients groups, but the frequency was low in the HIV-positive patients. In this group, $72 \%$ of samples were collected from asymptomatic subjects and influenza B infection was associated with the presence of symptoms.
In three of the 12 years of study, the predominant circulating lineage was different from that selected for vaccine composition. In 2002 and 2013, the influenza B component of the Southern Hemisphere influenza vaccine was of the $\mathrm{B} /$ Yamagata lineage, but more than $70 \%$ of the circulating viruses were identified as the $\mathrm{B} /$ Victoria lineage. Data from the Brazilian National Surveillance demonstrated that the Victoria-lineage reappeared in southern and southeastern Brazil during 2002 and predominated during the 2003, 2004, 2007, 2011 and 2012 influenza seasons. In contrast, the co-circulation of the two lineages was demonstrated during the 2002, 2005, 2006 and 2008 seasons (Motta et al. 2006, Paiva et al. 2013). In 2010, the B/Victoria lineage was recommended for the Southern Hemisphere vaccine; however, the vaccine that was distributed in Brazil was monovalent and included only the influenza A(H1N1)pdm09 strain. Data from international surveillance showed that, during the 2007-2008 influenza seasons, only $2 \%$ of the influenza $B$ viruses were identified as belonging to the $\mathrm{B} /$ Victoria lineage that was the influenza B component for the 2007-2008 Northern Hemisphere influenza vaccine (CDC 2008). During the 2011-2012 seasons in Taiwan, there was a large influenza B epidemic caused by a Yamagata lineage, but the influenza $B$ vaccine component was from the Victoria lineage (Lo et al. 2013).

Studies have shown that influenza-related direct and indirect costs were higher during the mismatched than matched seasons (Karve et al. 2013). Inclusion of the correct influenza B lineage in trivalent vaccines is a challenge; therefore, public health centres have analysed the possibility of reformulating the annual influenza vaccine to include both lineages of influenza $B$ viruses in a quadrivalent vaccine. Clinical trials with quadrivalent vaccines showed improved protection in children, were as safe and immunogenic as trivalent vaccines and could be an alternative to influenza vaccination (Domachowske et al. 2013, Greenberg et al. 2013).

A limitation of our study is the small number of positive samples that were analysed in some years, which may not represent the real proportion of circulating viruses. We were also not able to assess the real impact of match and mismatched seasons because the distribution of age groups was not homogeneous between studied years.

On the basis of data collected during a period of 12 years in a tertiary hospital of the largest city in the Southern Hemisphere, we found that teenagers were the most affected by influenza $\mathrm{B}$ infections, raising questions about which age groups should be included in vaccination programs. Co-circulation of both lineages was not common, but occurred recently. Furthermore, mismatch with the vaccine strain should be evaluated to support the introduction of a quadrivalent vaccine.

\section{REFERENCES}

Ambrose CS, Levin MJ 2012. The rationale for quadrivalent influenza vaccines. Hum Vaccin Immunother 8: 81-88.

Biere B, Bauer B, Schweiger B 2010. Differentiation of influenza B virus lineages Yamagata and Victoria by real-time PCR. J Clin Microbiol 48: 1425-1427.

CDC - Centers for Disease Control and Prevention 2008. Influenza activity - United States and worldwide, 2007-08 season. MMWR Morb Mortal Wkly Rep 57: 692-697. 
CDC - Centers for Disease Control and Prevention 2009. CDC protocol of real-time RT-PCR for influenza A(H1N1). Available from: who.int/csr/resources/publications/swineflu/CDCRealtimeRTPCR_SwineH1Assay-2009_20090430.pdf.

Chan PK, Chan MC, Cheung JL, Lee N, Leung TF, Yeung ACM, Wong MCS, Ngai KL, Nelson EAS, Hui DS 2013. Influenza B lineage circulation and hospitalization rates in a subtropical city, Hong Kong, 2000-2010. Clin Infect Dis 56: 677-684.

Chen R, Holmes EC 2008. The evolutionary dynamics of human influenza B virus. J Mol Evol 66: 655-663.

Domachowske JB, Pankow-Culot H, Bautista M, Feng Y, Claeys C, Peeters M, Innis BL, Jain V 2013. A randomized trial of candidate inactivated quadrivalent influenza vaccine versus trivalent influenza vaccines in children aged 3-17 years. J Infect Dis 207: 1878-1887.

Freitas FT 2013. Sentinel surveillance of influenza and other respiratory viruses, Brazil, 2000-2010. Braz J Infect Dis 17: 62-68.

Greenberg DP, Robertson CA, Noss MJ, Blatter MM, Biedenbender $\mathrm{R}$, Decker MD 2013. Safety and immunogenicity of a quadrivalent inactivated influenza vaccine compared to licensed trivalent inactivated influenza vaccines in adults. Vaccine 31: 770-776.

Gutiérrez-Pizarraya A, Pérez-Romero P, Alvarez R, Aydillo TA, Osorio-Gómez G, Milara-Ibáñez C, Sanchez M, Pachón J, Cordeiro E 2012. Unexpected severity of cases of influenza B infection in patients that required hospitalization during the first post-pandemic wave. $J$ Infect 65: 423-430.

Hite LK, Glezen WP, Demmler GJ 2007. Medically attended pediatric influenza during the resurgence of the Victoria lineage of influenza B virus. Int J Infect Dis 11: 40-47.
Karve S, Meier G, Davis KL, Misurski DA, Wang CC 2013. Influenza-related health care utilization and productivity losses during seasons with and without a match between the seasonal and vaccine virus B lineage. Vaccine 31: 3370-3388.

Lo YC, Chuang JH, Kuo HW, Huang WT, Hsu YF, Liu MT, Chen CH, Huang HH, Chang CH, Chou JH, Chang FY, Lin TY, Chiu WT 2013. Surveillance and vaccine effectiveness of an influenza epidemic predominated by vaccine-mismatched influenza B/Yamagata-lineage viruses in Taiwan, 2011-12 season. PLoS ONE 8: 1-7.

Motta FC, Siqueira MM, Lugon AK, Straliotto SM, Fernandes SB, Krawczuk MM 2006. The reappearance of Victoria lineage influenza B virus in Brazil, antigenic and molecular analysis. J Clin Virol 36: 208-214.

Olson DR, Heffernan RT, Paladini M, Konty K, Weiss D, Mostashari F 2007. Monitoring the impact of influenza by age: emergency department fever and respiratory complaint surveillance in New York City. PLoS Med 4: 1349-1361.

Paiva TM, Benega MA, Silva DB, Santos KC, Cruz AS, Hortenci MF, Barbieri MT, Monteiro MM, Barbosa HA, Carvalhanas TR 2013. Evolutionary pattern of reemerging influenza B/Victoria lineage viruses in São Paulo, Brazil, 1996-2012: implications for vaccine composition strategy. J Med Virol 85: 1983-1989.

Rota PA, Wallis TR, Harmon MW, Rota JS, Kendal AP, Nerome K 1990. Cocirculation of two distinct evolutionary lineages of influenza type B virus since 1983. Virology 175: 59-68.

Selvaraju SB, Selvarangan R 2010. Evaluation of three influenza A and B real-time reverse transcription-PCR assays and a new 2009 H1N1 assay for detection of influenza viruses. J Clin Microbiol 48: 3870. 